\title{
Bivariate Stochastic Modelling of Glucose and Insulin Regulatory System among Type -2 Diabetes Mellitus Patients
}

\author{
Kiran Kumar Paidipati ${ }^{1}$ and Tirupathi Rao Padi $^{2}$ \\ Dept. of Statistics, Pondicherry University, \\ Puducherry -605014, India. \\ 1'kirankumarpaidipati@gmail.com, ${ }^{2}$ drtrpadi@gmail.com
}

\begin{abstract}
Keywords: Stochastic Model, Glucose-Insulin Regulatory System, Simultaneous Linear Differential Equations
\end{abstract}

\begin{abstract}
In this paper, a bivariate stochastic model for glucose and insulin regulatory system is developed. Difference Differential equations are derived from the processes of arrival and consumption of glucose molecules and insulin granules in blood plasma. Probability generating functions and linear differential equations are obtained, through which statistical measures on glucose and insulin levels are derived. Numerical illustrations are provided with suitable sensitivity analysis. Model behaviour is analyzed while using the derived measures. Indicators on health care and management of Type-2 Diabetes can be obtained with this study.
\end{abstract}

\section{Introduction}

\subsection{Importance of the study:}

Glucose metabolism is the main source of getting energy to living cells. Normal and healthy human body has proper metabolism where the level of glucose shall be in the range of 70 to $110 \mathrm{mg} / \mathrm{dl}$ in the blood plasma. The changing patterns of glucose consumption in human body are observed due to several factors like sedentary life styles, obesity, ageing, etc. According to the studies of International Diabetes Federation, the most diabetic prone nations are china and India. Predictions of study speculate that India is going to occupy the first position with a prevalence of around 75.9 million people by 2035 (IDF Atlas $6^{\text {th }}$ Edition).

The system of Endocrine is playing a vital role in conversion of glucose into energy, which is normally referred as glucose metabolism. It includes a chemical reaction in the inner part of the cell with the combination of glucose, oxygen and water to release energy (ATP, Adenosine triphosphate), carbon-dioxide and water.

The mechanism of synchronized endocrine and digestive systems is to maintain the plasma glucose levels in the normal range. If the glucose levels of plasma in fasting is more than $110 \mathrm{mg} / \mathrm{dl}$ then the problem is called hyperglycemia where as if it is less than $70 \mathrm{mg} / \mathrm{dl}$ then the problem is referred as hypoglycemia. The person who is in either state is considered to be diabetic. It is cleared that the key role of insulin is to orderly maintenance of glucose levels in the blood plasma. Some organs are having the cells of nature that they require insulin to get the glucose through infusion technique. However, some organs of the body may utilize the glucose without involvement of insulin.

Glucose transporters are of two types, namely insulin independent and insulin dependent. Except brain and genital organs, all the other parts of the body require insulin to make use of glucose for getting energy to the cell. Among the healthy individuals, the minimum needed quantity of insulin will be produced by pancreas for glucose metabolism. However, insulin secretion depends on mass of beta cells in pancreas. The inferior functioning of pancreas and low quantum of insulin secretion are influenced by increased age, genetical history of diabetes, Sullen and sedentary life styles. Low levels of insulin causes to accumulation of glucose levels in the plasma and further leads to many health complications. 
Accumulation or consumption of glucose in blood stream is due to either adding or burning of calories. Food intake will increase the glucose whereas it is decreased through either physical activity of the body or extra physical exercise to the body. Conversion of glucose into energy depends on the availability and consumption of insulin. Usually insulin in the body will be available due to either after it is being secreted by pancreas or by means of inducing through external sources. The endocrine system in coordination with digestive system and glucose metabolism will regulate the levels of insulin based on the requirement blood glucose for various accounts of energy release. Failure of the above system's functioning results to imbalance of glucose and insulin, which is observed among the diabetic patients, particularly in Type- 2 diabetes.

This study is intended to understand the processes behind generation/utilization of glucose and secretion/ dispose of insulin along with regulating mechanism and metabolism processes of endocrine and digestive systems. It is assumed that arrivals and clearance processes of insulin as well as glucose are purely random in nature. Multi organ functioning on the regulating system of keeping the glucose and insulin in normal range is stochastic. Measuring the levels of both insulin and glucose through Markov point processes is the proposed work of this study.

\subsection{Reported studies:}

Mathematical biology, bio-statistical modeling of metabolism processes attracted the attention of many researchers. Several models based on the distributions of glucose and insulin has been developed with an objective of classical understanding of diabetes mechanism and its management.

Mathematical modeling of insulin-glucose dynamics became a breakthrough for assessment of effectiveness of glucose and sensitivity of insulin through its pioneering works with minimal and compartmental models [1, 2]. Insulin infusion models with respect to diabetes patients were developed with dynamics of glucose and insulin interactions [3]. Approximations of compartmental glucose kinetics of minimal models with Monte Carlo simulations revealed that Hidden Markov Models are more robust than minimal models [4]. Delayed-differential model to know the time course of glucose-insulin concentrations in an experimental data was developed as an extension of minimal models [5].

Physiological and pathological steady states with fixed points, the fastness of insulin and glucose dynamics are related to $\beta$-cell mass. Growth of beta cells mass indicates mild hyperglycemia considered to be negative feedback; contrary severe hyperglycemia indicates the low levels of beta cells considered to be positive feedback. Three pathways are extended to hyperglycemia includes physiological fixed point can be shifted to a hyperglycemia level; the physiological saddle point will balance the glucose levels and decreased glucose levels with progressive defects in glucose and/or insulin dynamics. Keeping the above notion, a model for the dynamics of glucose, insulin and mass of beta cells was developed with non linear differential equations [6]. The models in the forms of ordinary differential, partial differential, delayed differential and integro-differential equations were developed to study glucose-insulin regulatory system among diabetic patients [7]. Stochastic differential equations with continuous variable glucose levels were developed for studying the insulin dependent glucose consumption [8]. Simultaneous linear differential equations for Glucose, Insulin, Beta cell mass and Insulin receptor dynamics were developed for optimal management of diabetes [9].

\subsection{Motivation of study:}

Most of the mathematical models focused on the deterministic approach with certain physiological and biological assumptions related with diabetes mechanism with a focus of minimal models and compartmental models. The changing food and life styles with respect to diabetes patients make the levels of both insulin and glucose levels at more vulnerable stages. There is a need of assessing the quantum of glucose and insulin in plasma at a point of time through differential equations keeping 
the complexity of manual monitoring of glucose -insulin regulations. Due to many unexplained reasons, the levels of our interested variables (Glucose and Insulin quantity) are stochastic.

The primary arrival of glucose will be through food intake after proper transformation of carbohydrates or proteins or fats. Once blood plasma has received the glucose from hepatic porous system and if it is more than the normal range of healthy levels, then a partial quantum of it will be stored in liver as glycogen. Remaining levels of glucose will be transported to the needy cells with glucose transporters. Whenever there is a shortage of glucose levels (below the lower limit of normal range) in plasma, re arrival of glucose will be happened with liver as the stored glycogen will be converted into glucose with the help of glucagon from alpha cells. While consuming the glucose, body may use either insulin dependent glucose transporters or insulin independent glucose transporters.

Regarding the inflow and out flow of insulin is concerned, Insulin will be generated from beta cells in pancreas. The level of insulin secretion depends on the size of beta cells. If the mass of beta cells are sufficient to produce the required insulin, then the body will manage its activity with the available resources. Whereas the secreted insulin is not sufficient to meet the requirements of glucose conversion into energy, then external source of inducing insulin is required. Hence, the arrival of insulin is either with internal sources or with external induced sources. However, the insulin consumption is of two ways, the major part is with insulin dependent glucose transportation and the minor part of it is for conversion of glucose in to glycogen.

The total mechanism indicates that the accumulation and disposal of glucose molecules and insulin granules shall be viewed as discrete random processes. Their sizes are interdependent and regulating by some processes similar to arrivals and departure stochastic processes. The happening of arrivals, conversion and departure of glucose molecule; the arrival and departure of insulin granule are in simultaneous processes. This study proposed by using bivariate linear Poisson processes for deriving difference differential equations based on the assumptions of physiological/biological aspects related to glucose and insulin dynamics.

\section{Stochastic Model:}

The mechanism of food intake and its consumptions are heavily influenced by random factors. The following schematic diagram will explain the arrival and disposal of both Glucose and Insulin.
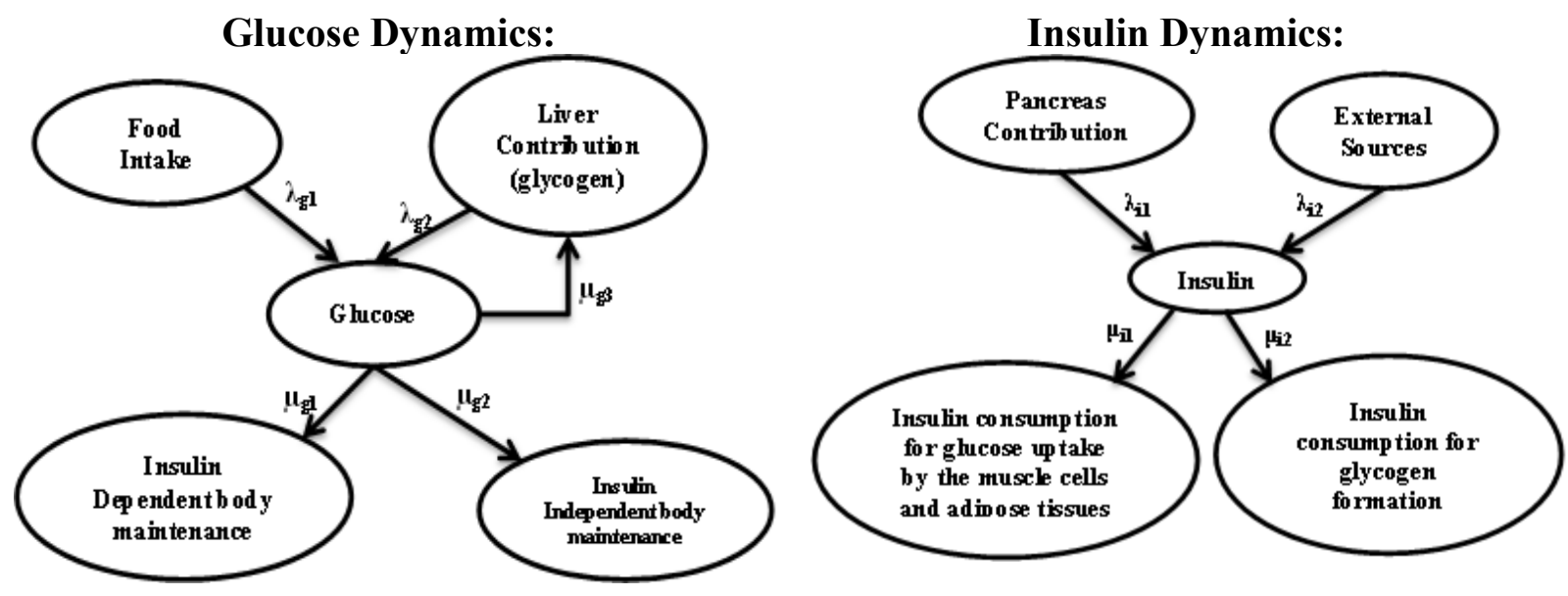

Figure -1: Schematic Diagram for Glucose and Insulin arrivals and disposals 


\subsection{Assumptions of the Model:}

Let $\lambda_{g 1}, \lambda_{g 2}$ be the arrival rates of glucose molecules to the blood plasma per unit time through food intake and liver respectively; $\mu_{g 1}, \mu_{g 2}$ and $\mu_{g 3}$ be the rates of glucose disposal per unit time due to insulin dependent cells, insulin independent cells and to transform glucose as glycogen respectively. Let $\lambda_{i 1}, \lambda_{i 2}$ be arrival rates of insulin granules per unit time from Pancreas and External sources respectively; $\mu_{i 1}, \mu_{i 2}$ be the disposal rates of insulin per unit time for the process of glucose absorption to the adipose tissues/muscle cells and for glucose conversion as glycogen respectively. Let $\mathrm{m}, \mathrm{n}$ are the concentrations of insulin and glucose at a point of time. Let ' $\mathrm{b}$ ' be the mass of beta cells. Let the processes of arrival and disposal of both glucose molecules and insulin granules follows Poisson distribution.

$P_{n, m}(\mathrm{t})$ be the probability of existing of ' $\mathrm{n}$ ' glucose molecules and ' $\mathrm{m}$ ' insulin granules in the blood stream at a point of time' $\mathrm{t}$ '.

$$
\begin{aligned}
P_{n, m}(\mathrm{t}+\Delta \mathrm{t})= & P_{n, m}(\mathrm{t})\left[1-\left(\lambda_{g 1} \Delta t+O(\Delta t)\right)\right] \\
& {\left[1-\left(\lambda_{g 2} \Delta t+O(\Delta t)\right)\right]\left[1-n m \mu_{g 1} \Delta t+O(\Delta t)\right] } \\
& {\left[1-n \mu_{g 2} \Delta t+O(\Delta t)\right]\left[1-n m \mu_{g 3} \Delta t+O(\Delta t)\right] } \\
& {\left[1-b \lambda_{i 1} \Delta t+O(\Delta t)\right]\left[1-\lambda_{i 2} \Delta t+O(\Delta t)\right] } \\
& {\left[1-n m \mu_{i 1} \Delta t+O(\Delta t)\right]\left[1-n m \mu_{i 2} \Delta t+O(\Delta t)\right] } \\
+ & P_{n-1, m}(t)\left\{\left[\lambda_{g 1} \Delta t+O(\Delta t)\right]+\left[\lambda_{g 2} \Delta t+O(\Delta t)\right]\right\} \\
+ & P_{n+1, m}(t)\left\{\left[n m \mu_{g 1} \Delta t+O(\Delta t)+\left[n \mu_{g 2} \Delta t+O(\Delta t)\right]+\left[n m \mu_{g 3} \Delta t+O(\Delta t)\right]\right.\right. \\
+ & P_{n, m-1}(t)\left\{\left[b \lambda_{i 1} \Delta t+O(\Delta t)\right]+\left[\lambda_{i 2} \Delta t+O(\Delta t)\right]\right\} \\
+ & P_{n, m+1}(t)\left\{\left[n m \mu_{i 1} \Delta t+O(\Delta t)\right]+\left[n m \mu_{i 2} \Delta t+O(\Delta t)\right]\right.
\end{aligned}
$$

Difference Differential equation is

$$
\begin{aligned}
\frac{d}{d t} P_{n, m}(\mathrm{t}) & =\left\{-\left(\lambda_{g 1}+\lambda_{g 2}+n m \mu_{g 1}+n \mu_{g 2}+n m \mu_{g 3}+b \lambda_{i 1}+\lambda_{i 2}+n m \mu_{i 1}+n m \mu_{i 2}\right)\right\} P_{n, m}(t) \\
+ & \left\{\left[\lambda_{g 1}+\lambda_{g 2}\right\} P_{n-1, m}(t)+\left\{(n+1) m \mu_{g 1}+(n+1) \mu_{g 2}\right.\right. \\
+ & \left.(n+1) m \mu_{g 3}\right\} P_{n+1, m}(t)+\left\{b \lambda_{i 1}+\lambda_{i 2}\right\} P_{n, m-1}(t) \\
+ & \left\{n(m+1) \mu_{i 1}+n(m+1) \mu_{i 2}\right\} P_{n, m+1}(t)
\end{aligned}
$$

The probability generating function is

$$
\begin{aligned}
P_{n, m}(x, y: \mathrm{t}) & =-\left(\lambda_{g 1}+\lambda_{g 2}\right) \sum_{n=0}^{\infty} \sum_{m=0}^{\infty} x^{n} y^{m} P_{n, m}(t)-\mu_{g 1} \sum_{n=0}^{\infty} \sum_{m=0}^{\infty} x^{n} y^{m} n m P_{n, m}(t) \\
& -\mu_{g 2} \sum_{n=0}^{\infty} \sum_{m=0}^{\infty} x^{n} y^{m} n P_{n, m}(t)-\mu_{g 3} \sum_{n=0}^{\infty} \sum_{m=0}^{\infty} x^{n} y^{m} n m P_{n, m}(t) \\
& -\left(b \lambda_{i 1}+\lambda_{i 2}\right) \sum_{n=0}^{\infty} \sum_{m=0}^{\infty} x^{n} y^{m} P_{n, m}(t)-\left(\mu_{i 1}+\mu_{i 2}\right) \sum_{n=0}^{\infty} \sum_{m=0}^{\infty} n m x^{n} y^{m} P_{n, m}(t) \\
& +\left\{\lambda_{g 1}+\lambda_{g 2}\right\} \sum_{n=0}^{\infty} \sum_{m=0}^{\infty} x^{n} y^{m} P_{n-1, m}(t)+\mu_{g 1} \sum_{n=0}^{\infty} \sum_{m=0}^{\infty} x^{n} y^{m}(n+1) m P_{n+1, m}(t) \\
& +\mu_{g 2} \sum_{n=0}^{\infty} \sum_{m=0}^{\infty}(n+1) x^{n} y^{m} P_{n+1, m}(t)+\mu_{g 3} \sum_{n=0}^{\infty} \sum_{m=0}^{\infty}(n+1) m x^{n} y^{m} P_{n+1, m}(t) \\
& +\left\{b \lambda_{i 1}+\lambda_{i 2}\right\} \sum_{n=0}^{\infty} \sum_{m=0}^{\infty} x^{n} y^{m} P_{n, m-1}(t)+\left(\mu_{i 1}+\mu_{i 2}\right) \sum_{n=0}^{\infty} \sum_{m=0}^{\infty} n(m+1) x^{n} y^{m} P_{n, m+1}(t)
\end{aligned}
$$


Partial differential equations with probability generating function is

$$
\begin{aligned}
\frac{\partial P(x, y ; t)}{\partial t}= & \left\{(x-1)\left(\lambda_{g 1}+\lambda_{g 2}\right)+(y-1)\left(b \lambda_{i 1}+\lambda_{i 2}\right)\right\} P(x, y ; t) \\
& +\left\{x y\left(\mu_{g 1}+\mu_{g 3}+\mu_{i 1}+\mu_{i 2}\right)+y\left(\mu_{g 1}+\mu_{g 3}\right)+x\left(\mu_{i 1}+\mu_{i 2}\right)\right\} \frac{\partial^{2} P(x, y ; t)}{\partial x \partial y} \\
& +\left\{(1-x) \mu_{g 2}\right\} \frac{\partial P(x, y ; t)}{\partial x}
\end{aligned}
$$

Partial differential equations with cumulant generating function is

$$
\begin{aligned}
\frac{\partial K(u, v ; t)}{\partial t}= & \left\{\left(u+\frac{u^{2}}{2}\right)\left(\lambda_{g 1}+\lambda_{g 2}\right)+\left(v+\frac{v^{2}}{2}\right)\left(b \lambda_{i 1}+\lambda_{i 2}\right)\right\}\left[u E_{x}+v E_{y}+\frac{u^{2}}{2} V_{x}+\frac{v^{2}}{2} V_{y}+u v E_{x y}\right] \\
& +\left\{-\left(\mu_{g 1}+\mu_{g 3}+\mu_{i 1}+\mu_{i 2}\right)+e^{-u}\left(\mu_{g 1}+\mu_{g 3}\right)+e^{-v}\left(\mu_{i 1}+\mu_{i 2}\right)\right\} \\
& {\left[E_{x y}-\left(\mathrm{E}_{x}+u V_{x}+v E_{x y}\right)-\left(\mathrm{E}_{y}+v V_{y}+u E_{x y}\right)-\left(u \mathrm{E}_{x}+v E_{y}+\frac{u^{2}}{2} V_{x}+\frac{v^{2}}{2} V_{y}+u v E_{x y}\right)\right] } \\
& +\left\{\left(1-e^{u}\right) \mu_{g 2}\right\}\left[\left(\mathrm{E}_{x}+u V_{x}+v E_{x y}\right)-\left(u \mathrm{E}_{x}+v E_{y}+\frac{u^{2}}{2} V_{x}+\frac{v^{2}}{2} V_{y}+u v E_{x y}\right)\right]
\end{aligned}
$$

On comparing the coefficients of $u^{\prime} s, v^{\prime} s, \frac{u^{2}}{2}{ }^{\prime} s,{\frac{v^{2}}{2}}^{\prime} s$ and $u v^{\prime} s$, arrived to the simultaneous ordinary differential equations.

\subsection{Differential Equations and Statistical Measures:}

Using the notion of probability generating and cumulant generating functions with partial derivatives with respect to ' $\mathrm{t}$ ', we have

$$
\begin{aligned}
& \frac{d}{d t}\left(E_{x}(t)\right)=-\left(\mu_{g 1}+\mu_{g 3}\right) \mathrm{E}_{x y}+\left[\mu_{g 1}+\mu_{g 3}-\mu_{g 2}\right] \mathrm{E}_{x}+\left[\mu_{g 1}+\mu_{g 3}\right] \mathrm{E}_{y} \\
& \frac{d}{d t}\left(E_{y}(t)\right)=-\left(\mu_{i 1}+\mu_{i 2}\right) \mathrm{E}_{x y}+\left(\mu_{i 1}+\mu_{i 2}\right) \mathrm{E}_{x}+\left(\mu_{i 1}+\mu_{i 2}\right) E_{y} \\
& \frac{d}{d t}\left(V_{x}(t)\right)=\left[2\left(\lambda_{g 1}+\lambda_{g 2}\right)+\left(\mu_{g 1}+3 \mu_{g 2}+\mu_{g 3}\right)\right] \mathrm{E}_{x}+\left[2\left(\mu_{g 1}+\mu_{g 3}-\mu_{g 2}\right)\right] \mathrm{V}_{x} \\
& \quad+3\left(\mu_{g 1}+\mu_{g 3}\right) \mathrm{E}_{x y}-\left(\mu_{g 1}+\mu_{g 3}\right) \mathrm{E}_{y} \\
& \frac{d}{d t}\left(V_{y}(t)\right)=\left[2\left(b \lambda_{i 1}+\lambda_{i 2}\right)+\left(\mu_{i 1}+\mu_{i 2}\right)\right] \mathrm{E}_{y} \\
& \quad+2\left(\mu_{i 1}+\mu_{i 2}\right) \mathrm{V}_{y}-\left(\mu_{i 1}+\mu_{i 2}\right) \mathrm{E}_{x}+\left(\mu_{i 1}+\mu_{i 2}\right) \mathrm{E}_{x y} \\
& \frac{d}{d t}\left(E_{x y}(t)\right)=\left[\left(\lambda_{g 1}+\lambda_{g 2}\right)+\left(\mu_{g 1}+\mu_{g 2}+\mu_{g 3}\right)\right] \mathrm{E}_{y} \\
& +\left[\left(b \lambda_{i 1}+\lambda_{i 2}\right)+\left(\mu_{i 1}+\mu_{i 2}\right)\right] \mathrm{E}_{x}+\left(\mu_{g 1}+\mu_{g 3}\right) \mathrm{V}_{y} \\
& +\left(\mu_{i 1}+\mu_{i 2}\right) \mathrm{V}_{x}+\left[\left(\mu_{g 1}+\mu_{g 3}\right)+\left(\mu_{i 1}+\mu_{i 2}\right)-\mu_{g 2}\right] \mathrm{E}_{x y}
\end{aligned}
$$

where $E_{x}(t)$ is average level of glucose at time ' $\mathrm{t}$ '; $E_{y}(t)$ is average level of insulin at time 't'; $V_{x}(t)$ is the variance of glucose level at time ' $\mathrm{t}$ '; $V_{y}(t)$ is the variance of insulin level at time ' $\mathrm{t}$ ' $E_{x y}(t)$ is average of glucose and insulin levels at time ' $\mathrm{t}$ '.

By considering

$$
\begin{aligned}
& a_{1}=\left(\mu_{g 1}+\mu_{g 3}\right) ; \quad a_{2}=\left(\mu_{g 1}+\mu_{g 3}-\mu_{g 2}\right) ; \quad a_{3}=\mu_{i 1}+\mu_{i 2} ; \quad a_{4}=2\left(\lambda_{g 1}+\lambda_{g 2}\right)+\left(\mu_{g 1}+3 \mu_{g 2}+\mu_{g 3}\right) ; \\
& a_{5}=2\left(b \lambda_{i 1}+\lambda_{i 2}\right)+\left(\mu_{i 1}+\mu_{i 2}\right) ;
\end{aligned}
$$




$$
\begin{aligned}
& a_{6}=\left(\lambda_{g 1}+\lambda_{g 2}\right)+\left(\mu_{g 1}+\mu_{g 2}+\mu_{g 3}\right) ; a_{7}=\left(b \lambda_{i 1}+\lambda_{i 2}\right)+\left(\mu_{i 1}+\mu_{i 2}\right) ; \\
& a_{8}=\mu_{g 1}+\mu_{g 3}+\left(\mu_{i 1}+\mu_{i 2}\right)-\mu_{g 2} ; b_{1}=\frac{\left(2 a_{2} a_{3} V_{x}-6 a_{1} a_{3} V_{y}\right)}{a_{1}\left(a_{3}+3 a_{5}\right)} ; b_{2}=\frac{\left(a_{3} a_{4}+3 a_{1} a_{3}\right)}{a_{1}\left(a_{3}+3 a_{5}\right)} ; \\
& b_{3}=\frac{\left(2 a_{2} V_{x}-a_{1} b_{1}\right)}{3 a_{1}} ; b_{4}=\frac{\left(a_{4}-a_{1} b_{2}\right)}{3 a_{1}} ; d_{1}=\frac{\left(a_{1} b_{1}-a_{1} b_{3}\right)}{\left(a_{2}+a_{1} b_{4}+a_{1} b_{2}\right)} ; d_{2}=G_{0}+\frac{\left(a_{1} b_{1}-a_{1} b_{3}\right)}{\left(a_{2}+a_{1} b_{4}+a_{1} b_{2}\right)} ; \\
& d_{3}=-a_{3} b_{3}+\left(a_{3} b_{4}+a_{3}\right) \mathrm{d}_{1} ; \alpha=a_{2}+a_{1} b_{4}+a_{1} b_{2} ; H_{1}=a_{7} d_{2}-a_{6} d_{2}\left(1+b_{4}\right) ; \\
& H_{2}=\left(a_{7} d_{1}+a_{1} V_{y}+a_{3} V_{x}-a_{6} \frac{d_{3}}{a_{3}}\right) ; k=I_{0}+\frac{d_{3}}{a_{3}}+d_{2}\left(1+b_{4}\right) ;
\end{aligned}
$$

$\mathrm{G}_{0}$ and $\mathrm{I}_{0}$ are the initial sizes of glucose and insulin respectively. Solving the set of linear simultaneous differential equations from (2.2.1) to (2.2.5), we will get the following measures by fixing variability of glucose and insulin levels.

Average number of glucose molecules in the blood stream is

$$
\mathrm{E}_{x}(t)=d_{1}+e^{\alpha t} d_{2}
$$

Average number of insulin granules in the blood stream is

$$
E_{y}(t)=-\frac{d_{3}}{a_{3}}-d_{2}\left(1+b_{4}\right) e^{\alpha t}+k e^{a_{3} t}
$$

Covariance of glucose molecules and insulin granules is

$$
\operatorname{Cov}_{x y}(t)=\frac{H_{1} e^{\alpha t}}{\left(\alpha-a_{8}\right)}+\frac{a_{6} k e^{a_{3} t}}{\left(a_{3}-a_{8}\right)}-\frac{H_{2}}{a_{8}}+p \cdot e^{a_{8} t}+\left(d_{1}+e^{\alpha t} d_{2}\right)\left(\frac{d_{3}}{a_{3}}+d_{2}\left(1+b_{4}\right) e^{\alpha t}-k e^{a_{3} t}\right)
$$

\subsection{Numerical Illustration and Finding:}

In order to measure the glucose and insulin levels with the developed model, a hypothetical numerical data set was generated for values of average levels of glucose, average level of insulin at a point of time and the covariance between the levels of glucose and insulin for changing values of one parameter when other parameters are constants. The results were presented in Table-1 of annexure-I. Graphical presentations were also given for better understanding of the influencing variables with the respective independent variables.
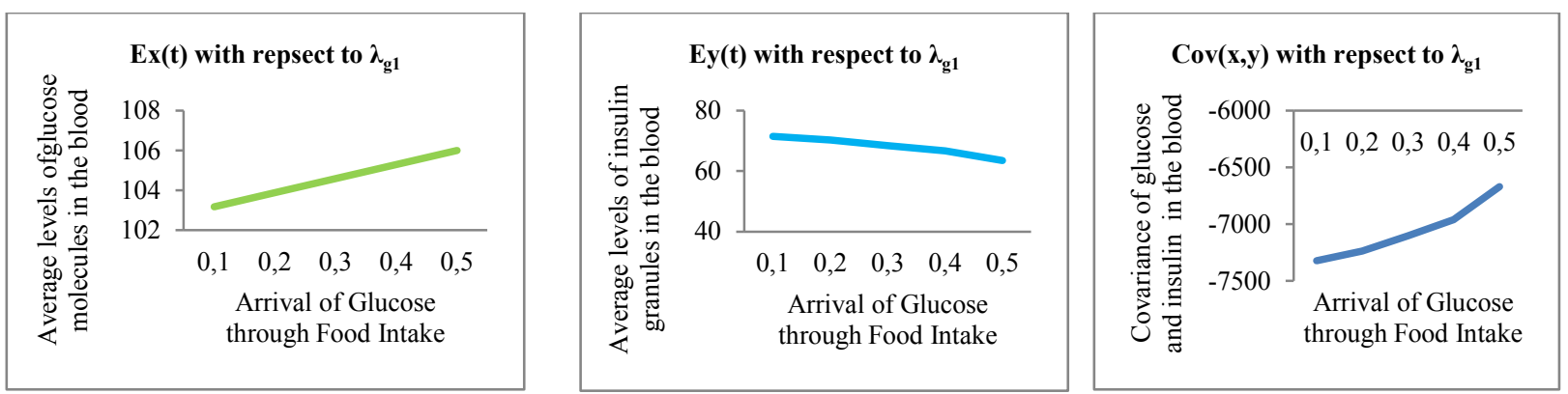

It is observed that $E_{x}(t)$ is an increasing function and $E_{y}(t)$ is decreasing function of $\lambda_{g 1}$ when the other parameters are constants. It may implies that there is (i) Positive relation between rate of arrival of glucose from food intake and the average levels of glucose in the blood plasma; (ii) Negative relation between rate of arrivals of glucose through food intake and the average levels of insulin in the blood stream; (iii) Further it may implies that the covariance of average levels of glucose molecules and average levels of insulin granules is negative and increasing function with respect to the rate of arrivals of glucose through food intake. 

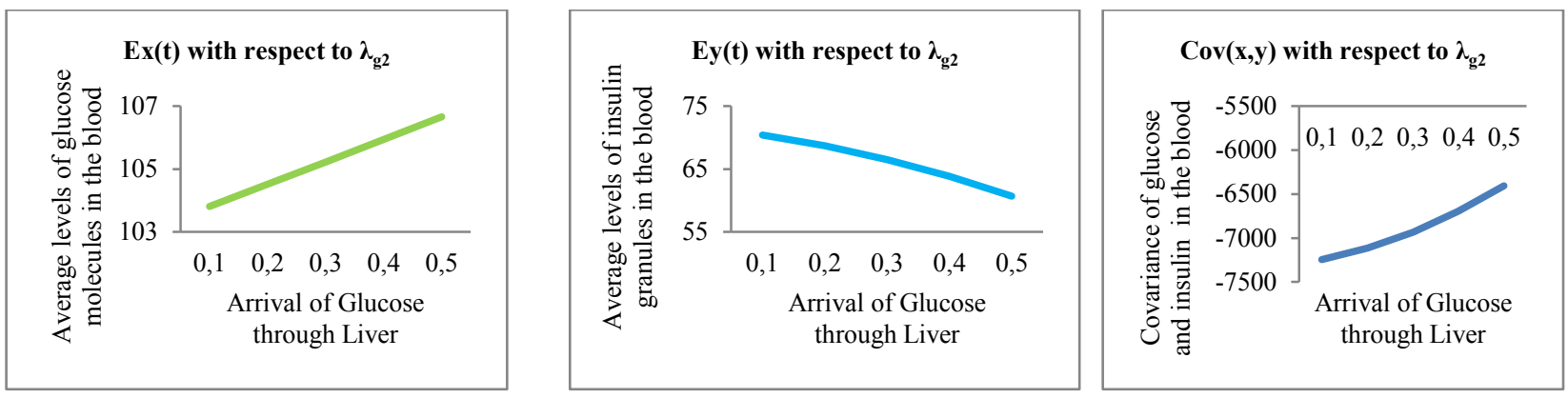

It is observed that $E_{x}(t)$ is an increasing function and $E_{y}(t)$ is decreasing function of $\lambda_{g 2}$ when the other parameters are constants. It may implies that there is (i) Positive relation between rate of arrival of glucose through liver and the average levels of glucose in the blood stream. (ii) Negative relation between rate of arrivals of glucose through liver and the average levels of insulin in the blood stream. (iii)The covariance of average levels of glucose and average levels of insulin is negative and increasing function with respect to the rate of arrivals of glucose through liver.
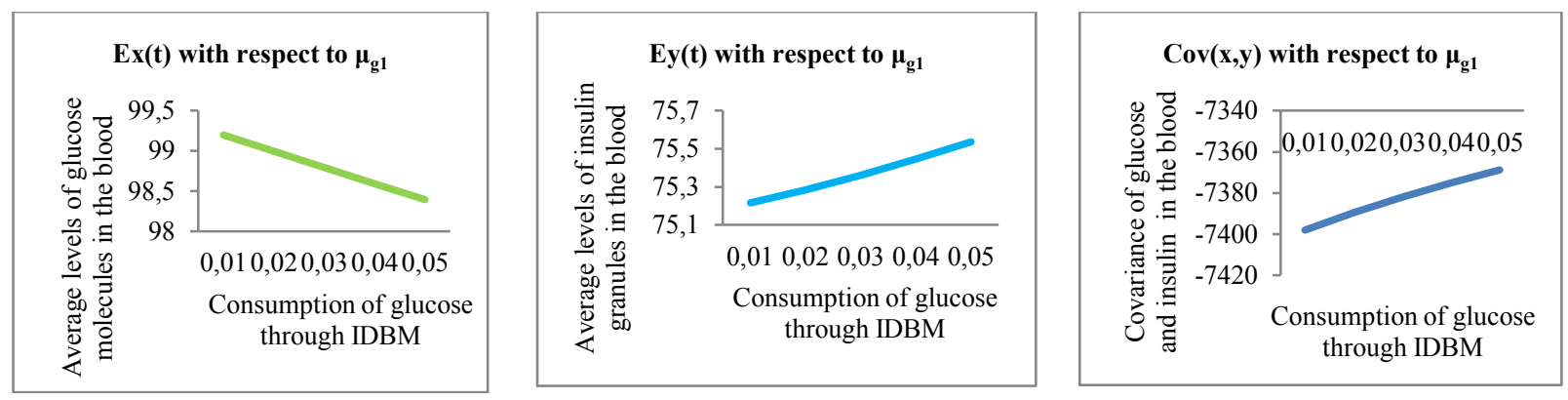

It is observed that $E_{x}(t)$ is decreasing function and $E_{y}(t)$ is an increasing function of $\mu_{g 1}$ when the other parameters are constants. It may implies that there is (i)Negative relation between rate of consumption of glucose through Insulin Dependent Body Maintenance (IDBM) and the average levels of glucose in the blood stream. (ii) Positive relation between rate of consumption of glucose through Insulin Dependent Body Maintenance (IDBM) and the average levels of insulin in the blood stream. (iii) The covariance of average levels of glucose and average levels of insulin is negative and increasing function with respect to the rate of consumption of glucose through Insulin Dependent Body Maintenance (IDBM).
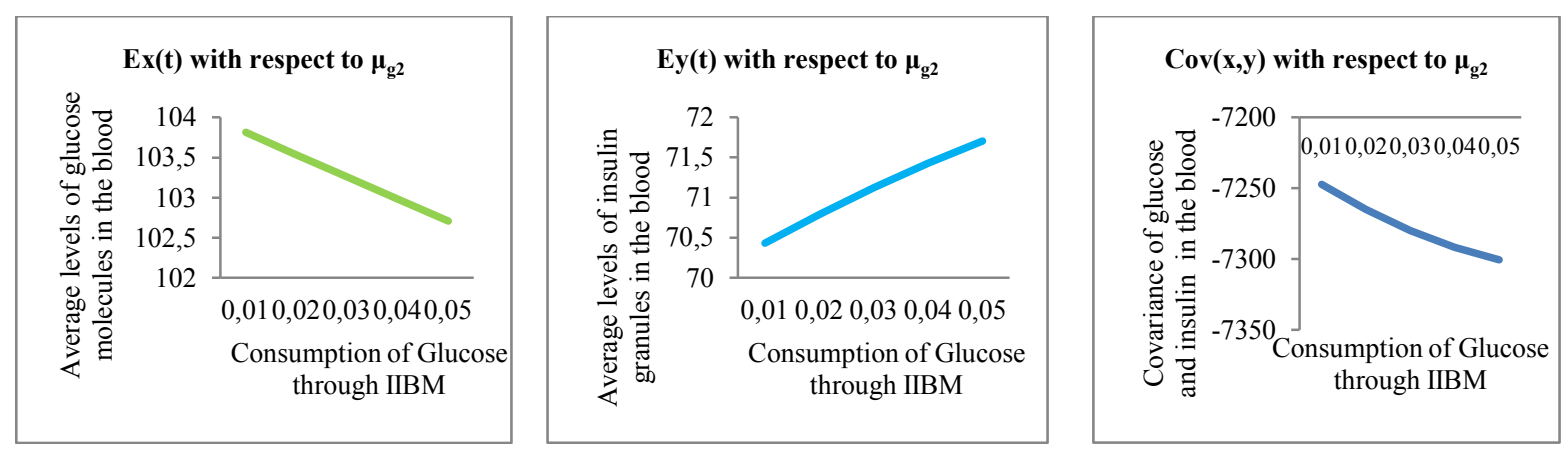

It is observed that $E_{x}(t)$ is decreasing function and $E_{y}(t)$ is an increasing function of $\mu_{g 2}$ when the other parameters are constants. It may implies that there is (i) Negative relation between rate of consumption of glucose through Insulin Independent Body Maintenance (IIBM) and the average levels of glucose in the blood stream. (ii) Positive relation between rate of consumption of glucose through Insulin Independent Body Maintenance (IIBM) and the average levels of insulin in the blood stream. (iii)The covariance of average levels of glucose and average levels of insulin is 
negative and increasing function with respect to the rate of consumption of glucose through Insulin Dependent Body Maintenance (IIBM).
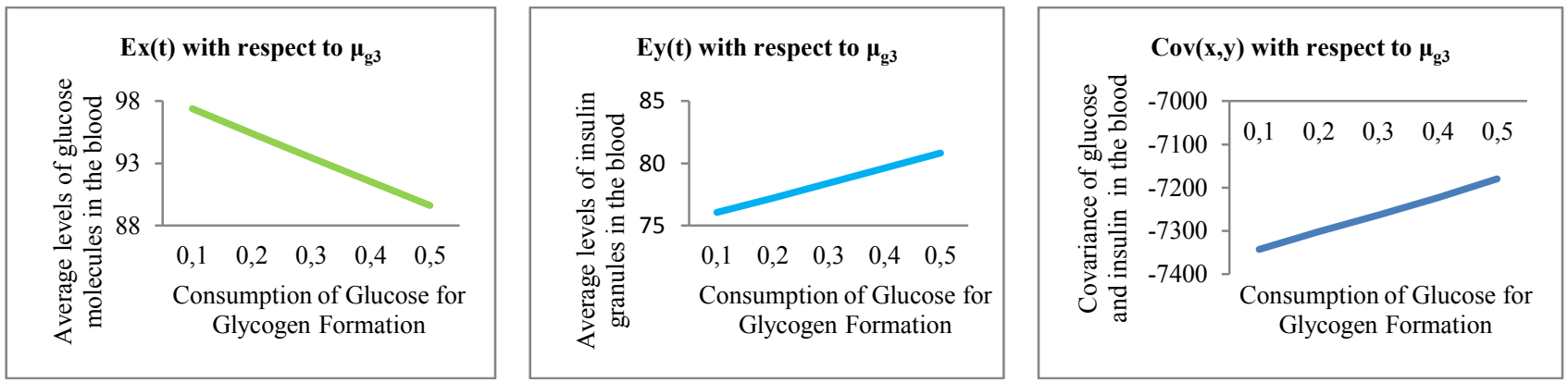

It is observed that $E_{x}(t)$ is decreasing function and $E_{y}(t)$ is an increasing function of $\mu_{g 3}$ when the other parameters are constants. It may implies that there is (i) Negative relation between rate of consumption of glucose for Glycogen formation and the average levels of glucose in the blood stream. (ii) Positive relation between rate of consumption of glucose for Glycogen formation and the average levels of insulin in the blood stream. (iii) The covariance of average levels of glucose and average levels of insulin is negative and increasing function with respect to the rate of consumption of converting the glucose as Glycogen formation.
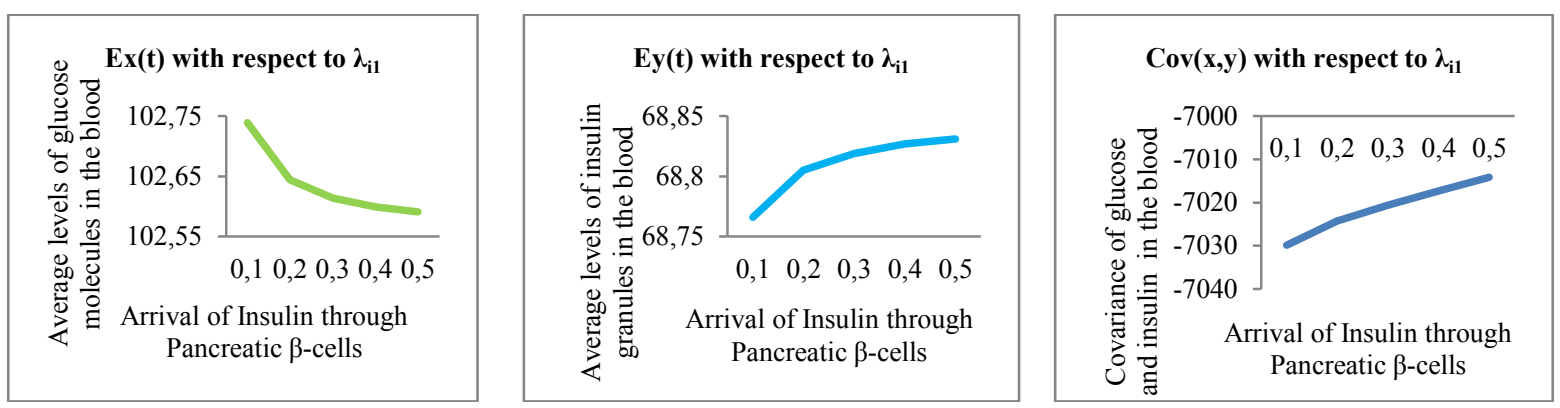

It is we observed that $E_{x}(t)$ is decreasing function and $E_{y}(t)$ is an increasing function of $\lambda_{i 1}$ when the other parameters are constants. It may imply that there is (i) Negative relation between rate of secretion of insulin from Pancreatic $\beta$-cells and the average levels of glucose in the blood stream. (ii) Positive relation between rates of secretion of insulin from Pancreatic $\beta$-cells and the average levels of insulin in the blood stream. (iii) The covariance of average levels of glucose and average levels of insulin is negative and increasing function with respect to the rate of secretion of insulin from Pancreatic $\beta$-cells.
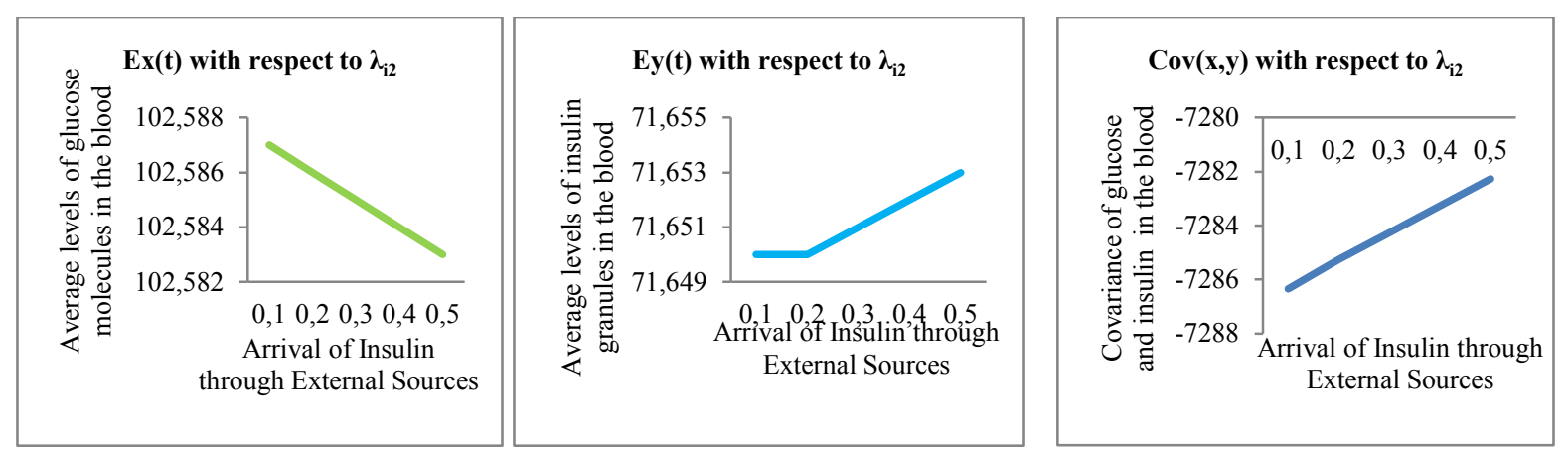

It is observed that $E_{x}(t)$ is decreasing function and $E_{y}(t)$ is an increasing function of $\lambda_{i 2}$ when the other parameters are constants. It may implies that there is (i) Negative relation between rate of arrival of insulin from External sources and the average levels of glucose in the blood stream. (ii) 
Positive relation between rate of arrival of insulin from External sources and the average levels of insulin in the blood stream. (iii) The covariance of average levels of glucose and average levels of insulin is negative and increasing function with respect to the rate of arrival of insulin from External sources.
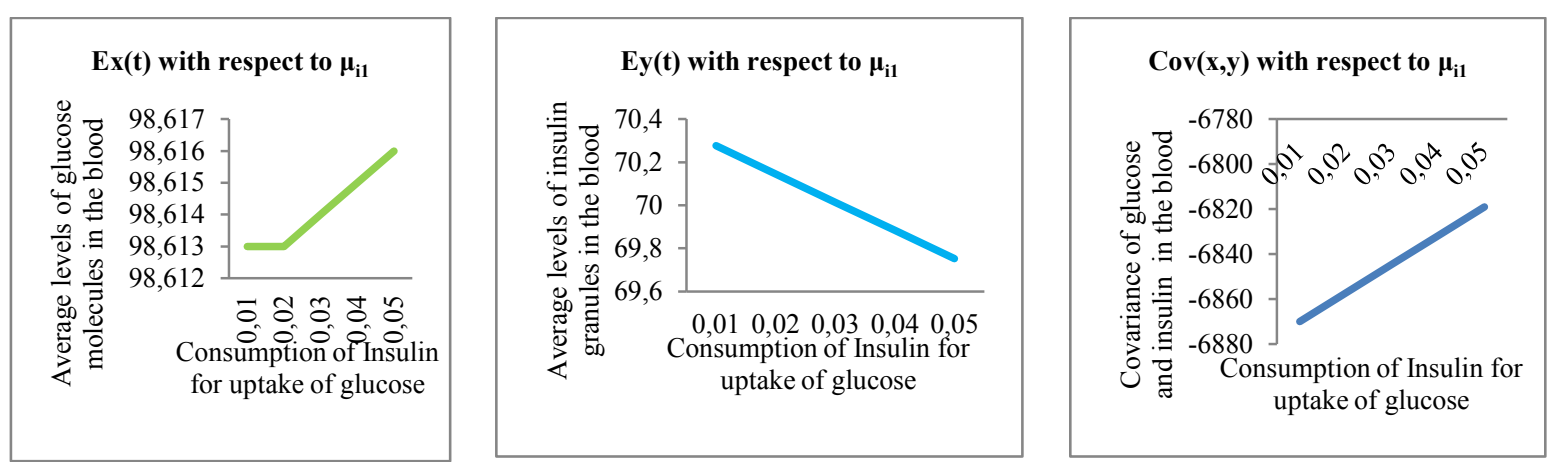

It is observed that $E_{x}(t)$ is an increasing function and $E_{y}(t)$ is decreasing function of $\mu_{i 1}$ when the other parameters are constants. It may implies that there is (i) Positive relation between rate of consumption of insulin for glucose absorption to the muscle cells and adipose tissues and the average levels of glucose in the blood stream. (ii) Negative relation between rate of consumption of insulin for glucose absorption to the muscle cells and adipose tissues and the average levels of insulin in the blood stream. (iii) The covariance between average levels of glucose and average levels of insulin is negative and increasing function with respect to the rate of consumption of insulin for glucose absorption to the muscle cells and adipose tissues.
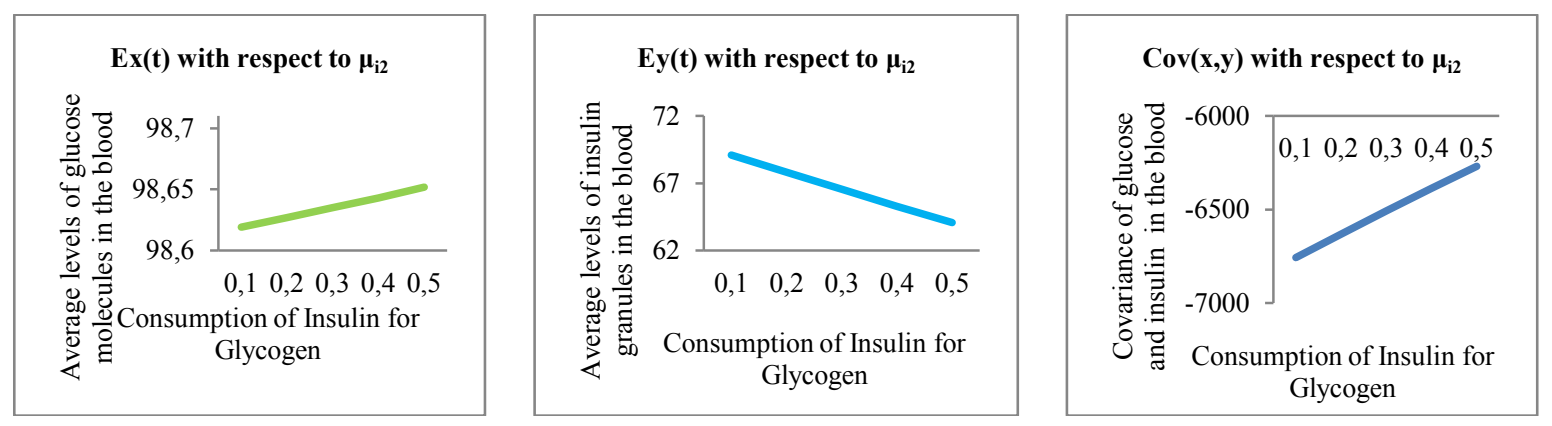

It is observed that $E_{x}(t)$ is an increasing function and $E_{y}(t)$ is decreasing function of $\mu_{i 2}$ when the other parameters are constants. It may implies that there is (i) Positive relation between rate of consumption of insulin for converting the glucose as glycogen and the average levels of glucose in the blood stream. (ii) Negative relation between rate of consumption of insulin for converting the glucose as glycogen and the average levels of insulin in the blood stream. (iii) The covariance between average levels of glucose and average levels of insulin is negative and increasing function with respect to the rate of consumption of insulin for converting the glucose as glycogen.
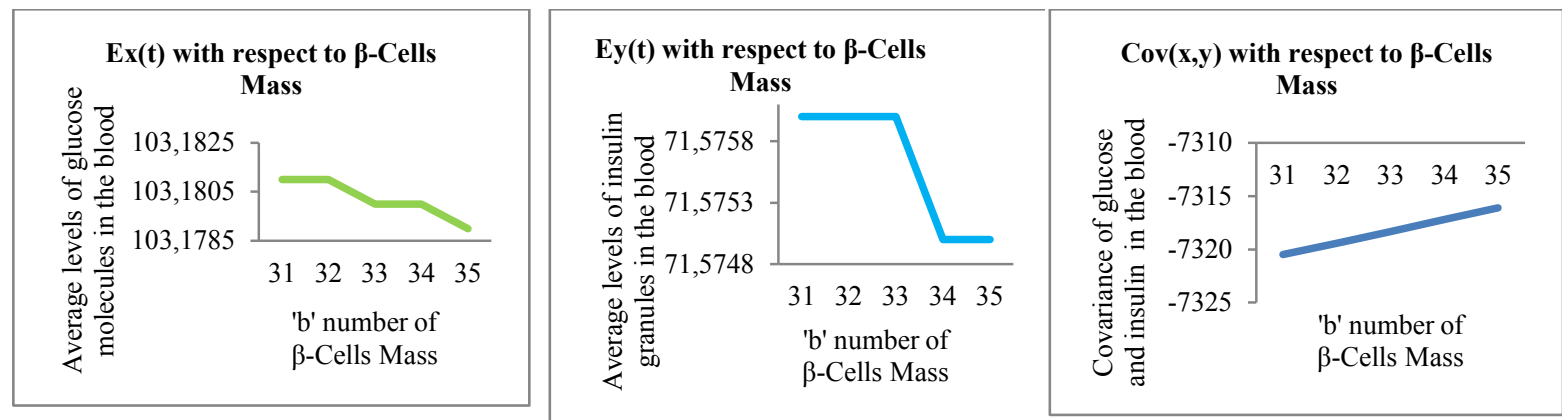
It is observed that $E_{x}(t)$ and $E_{y}(t)$ are decreasing functions of $\beta$-cell mass when the other parameters are constants. It may implies that there is (i) Negative relation between rate of increasing insulin secretion from the pancreatic $\beta$-cell mass and the average levels of glucose in the blood stream. (ii) Negative relation between rate increasing insulin secretion from the pancreatic $\beta$-cell mass and the average levels of insulin in the blood stream it may be because of simultaneous insulin consumption in the process of glucose metabolism. (iii) The covariance between the average levels of glucose and average levels of insulin is negative and increasing function with respect to the rate of increasing insulin secretion from the pancreatic $\beta$-cell mass.
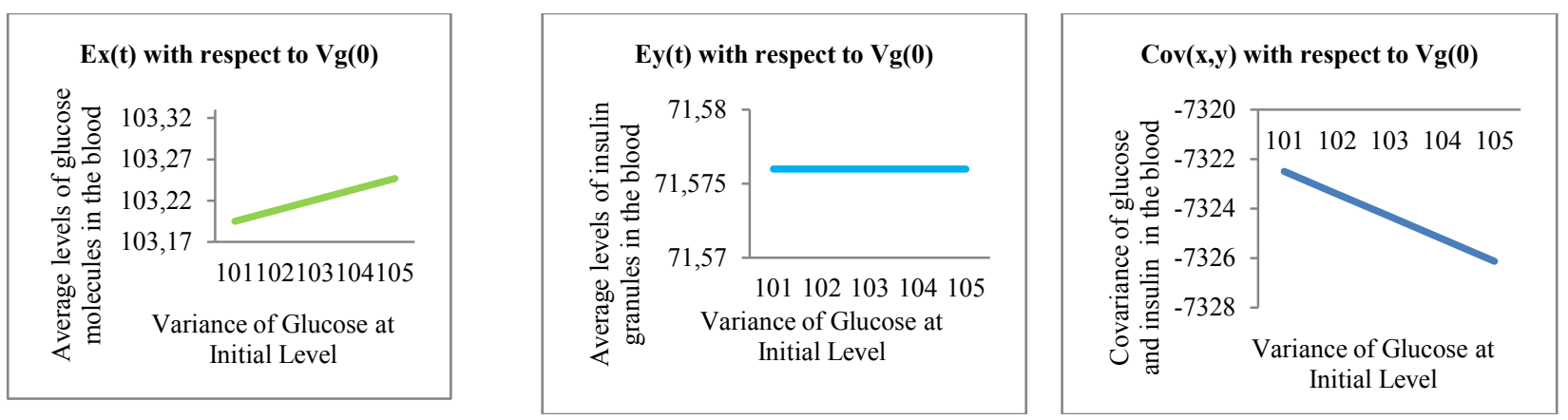

It is observed that $E_{x}(t)$ is an increasing function and $E_{y}(t)$ is invariant function of $V_{g}(0)$ when the other parameters are constants. It may implies that there is (i) Positive relation between the variance of glucose at initial level and the average levels of glucose in the blood stream. (ii) The invariant relation between the variance of glucose at initial level and the average levels of insulin in the blood stream. (iii) The covariance between the average levels of glucose and average levels of insulin is negative and decreasing function with respect to the variance of glucose at initial level.
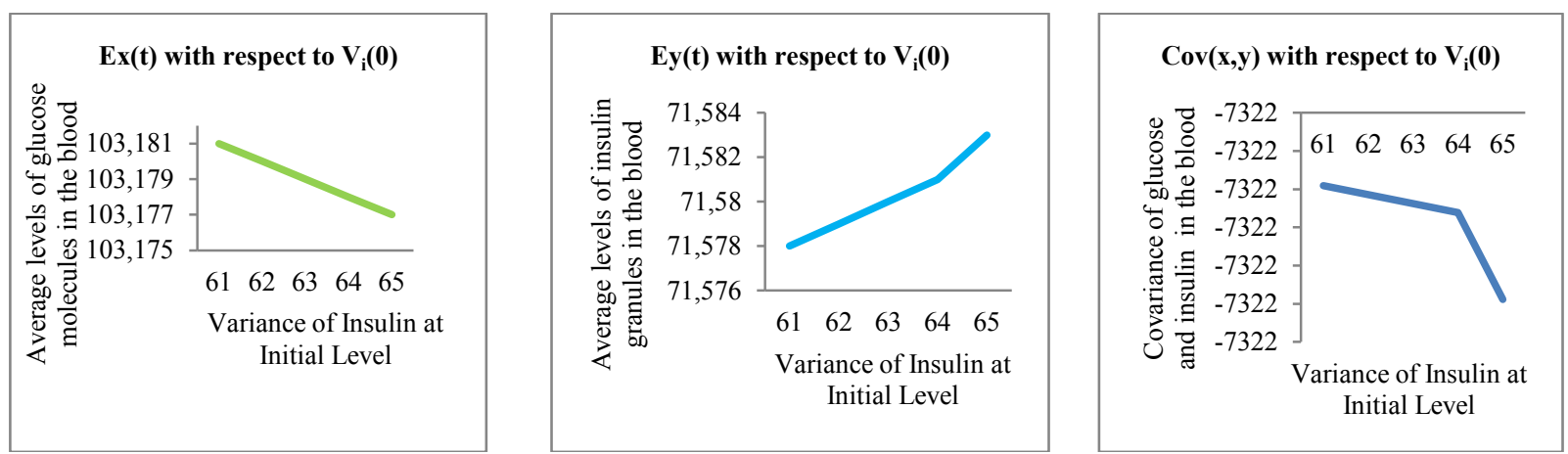

It is observed that $E_{x}(t)$ is decreasing function and $E_{y}(t)$ is an increasing function of $V_{i}(0)$ when the other parameters are constants. It may imply that there is (i) Negative relation between the variance of insulin at initial level and the average levels of glucose in the blood stream. (ii) Positive relation between the variance of insulin at initial level and the average levels of insulin in the blood stream. (iii) The covariance between average levels of glucose and average levels of insulin is negative and remain invariant with respect to the variance of insulin at initial level.
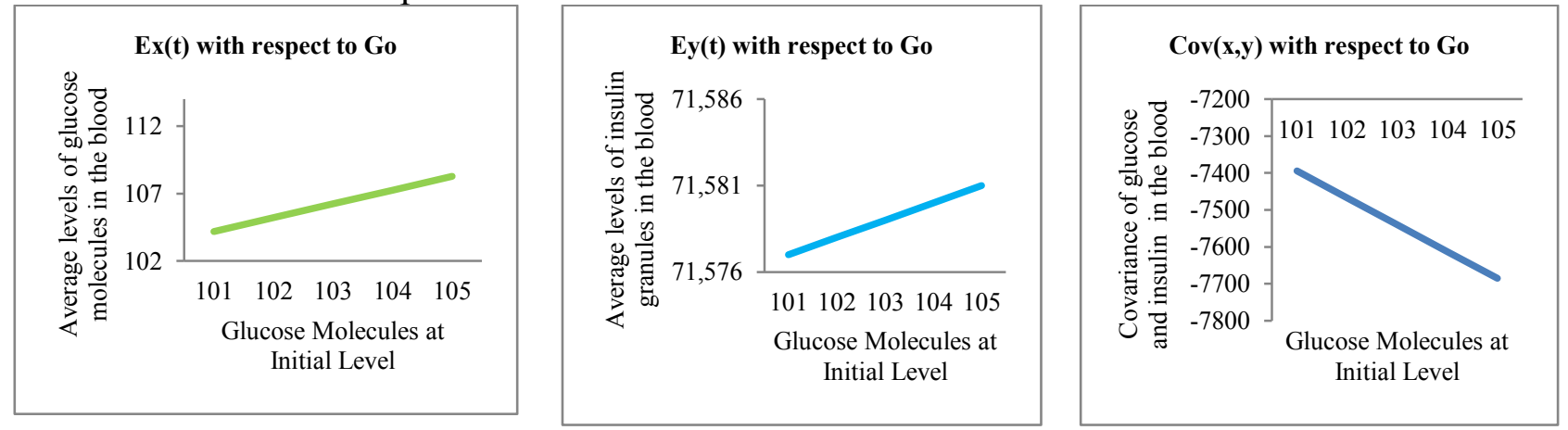
It is observed that $E_{x}(t)$ is an increasing function and $E_{y}(t)$ is increasing function of $G_{0}$ when the other parameters are constants. It may implies that there is (i) Positive relation between the glucose at initial level and the average levels of glucose in the blood stream. (ii) Positive relation between the glucose at initial level and the average levels of insulin in the blood stream. (iii) The covariance between average levels of glucose and average levels of insulin is negative and decreasing function with respect to the glucose at initial level.
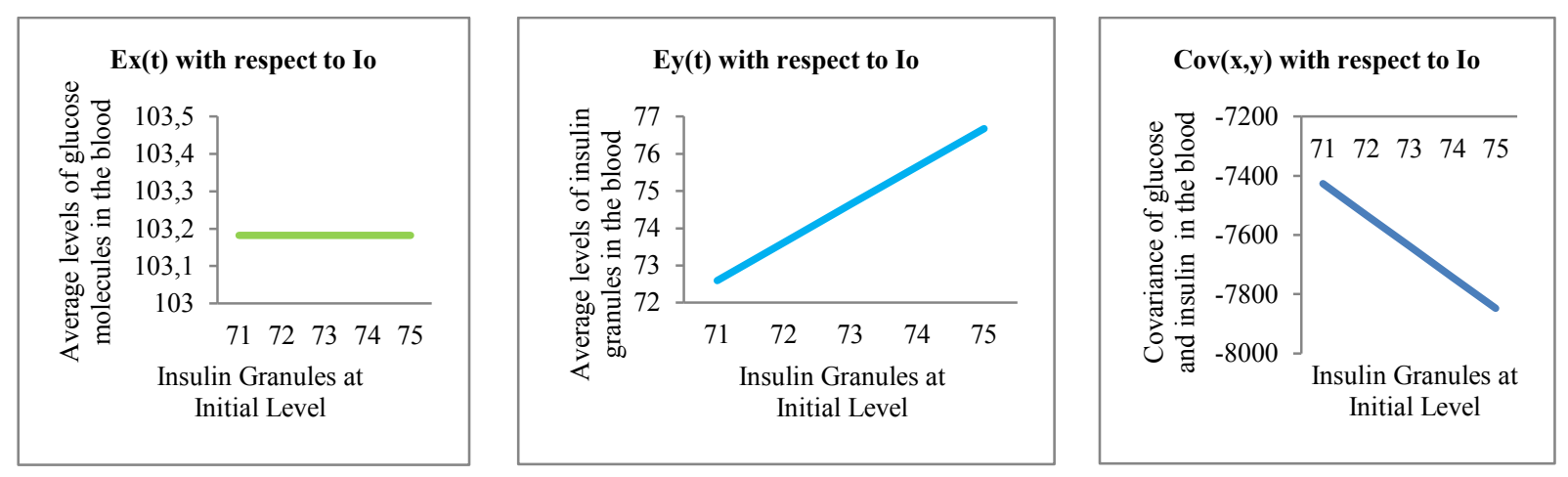

It is observed that $E_{x}(t)$ is an invariant function and $E_{y}(t)$ is an increasing function of $I_{0}$ when the other parameters are constants. It may implies that there is (i) No relation between insulin at initial level and the average levels of glucose in the blood stream. (ii) Positive relation between insulin at initial level and the average levels of insulin in the blood stream. (iii) The covariance between average levels of glucose and average levels of insulin is negative and decreasing function with respect to the insulin at initial level.
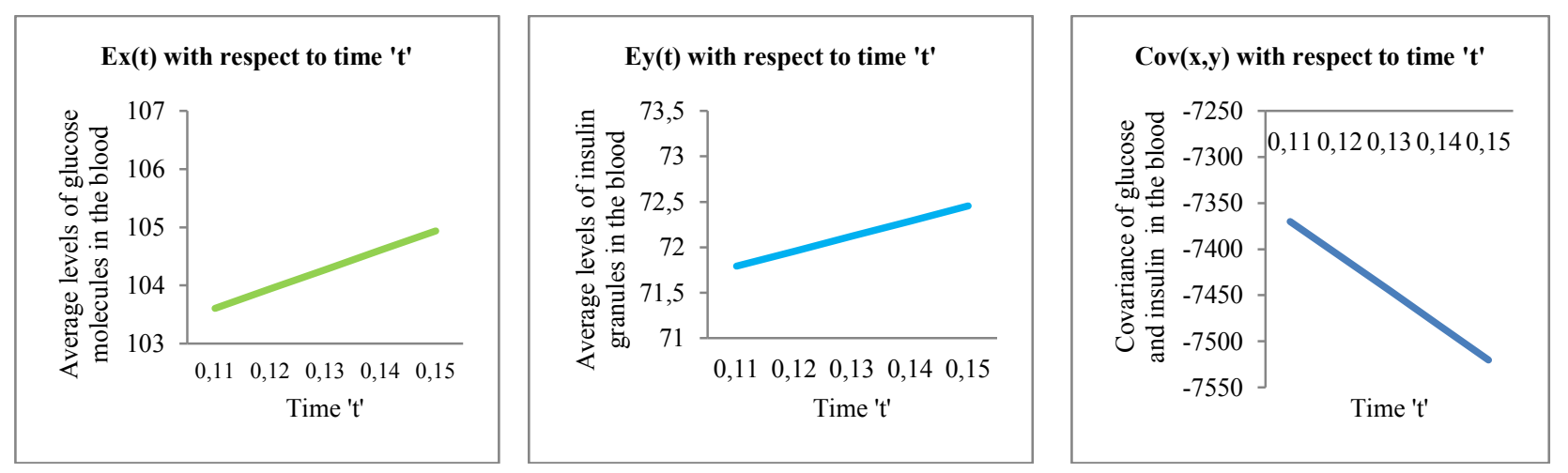

It is observed that $E_{x}(t)$ and $E_{y}(t)$ are increasing functions of Time' $t^{\prime}$ when the other parameters are constants. It may implies that there is (i) Positive relation between the time ' $t$ ' and the average levels of glucose in the blood stream during glucose arrival time. (ii) Positive relation between the time ' $t$ ' and the average levels of insulin in the blood stream during glucose arrival time. (iii) The covariance between average levels of glucose and average levels of insulin is negative and decreasing function of time' $t$ '. 


\section{Annexure -I}

Table -1: Values of Average levels of Glucose, Average level of Insulin at a point of time and the covariance between the levels of glucose and insulin for changing values of one parameter when other parameters are constants

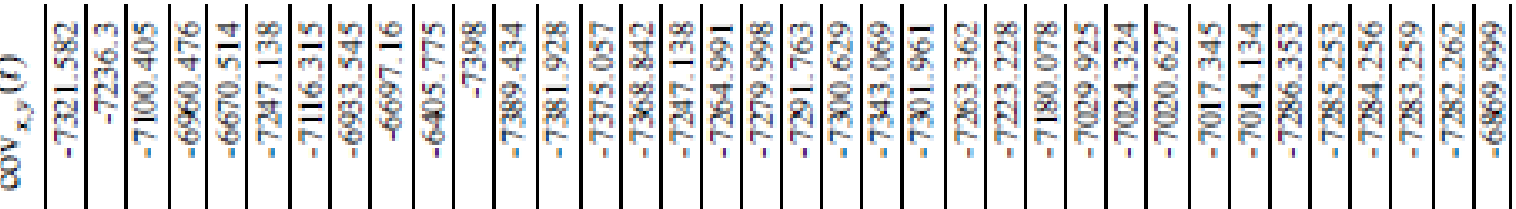

3
5

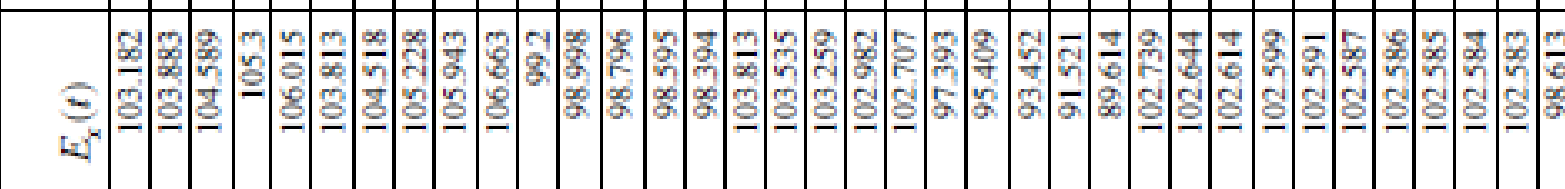

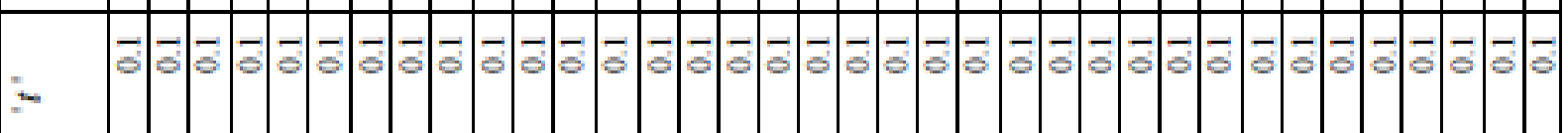

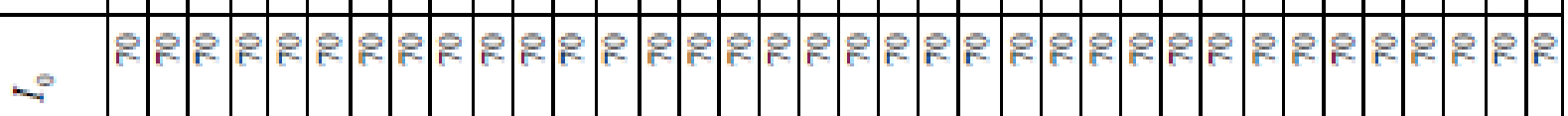

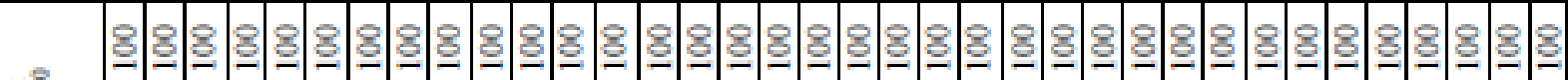

$0^{\circ}$

(2)

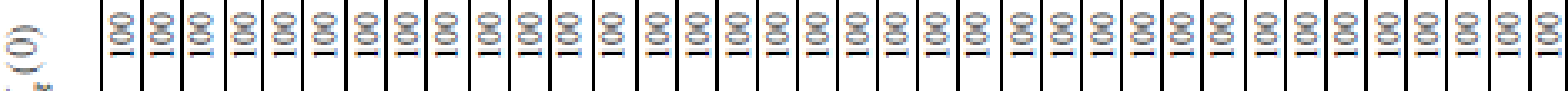

$i^{\infty}$

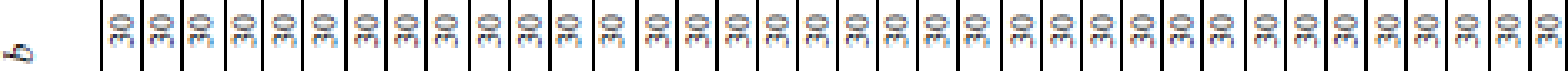

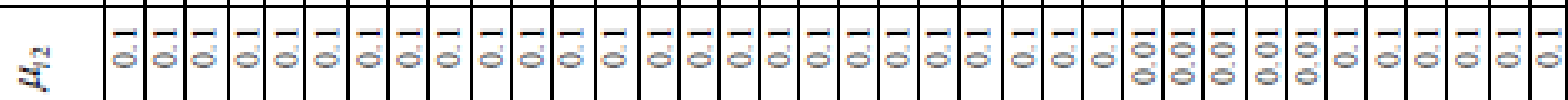

5- - - - - - - - - - - - - - - - - - - - - - - - - - - - - -

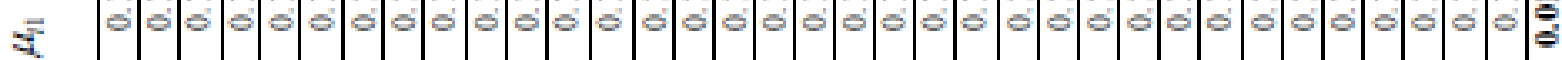

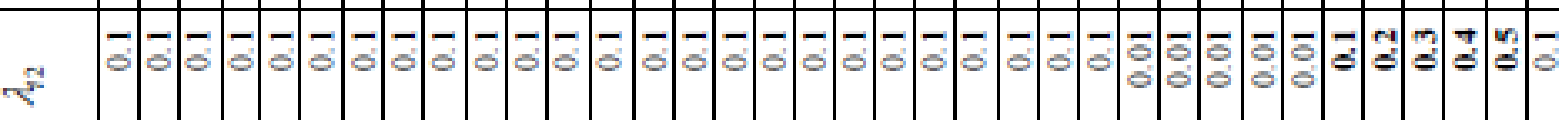

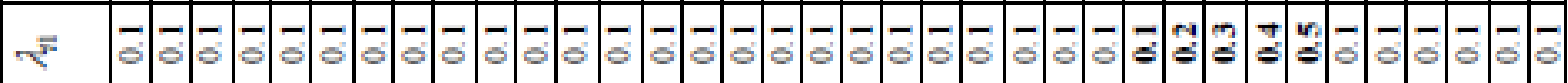

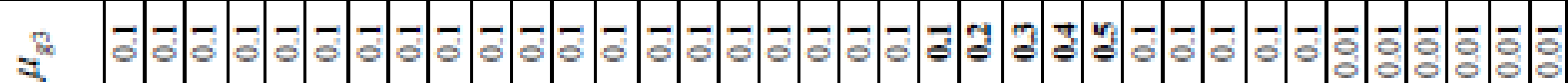

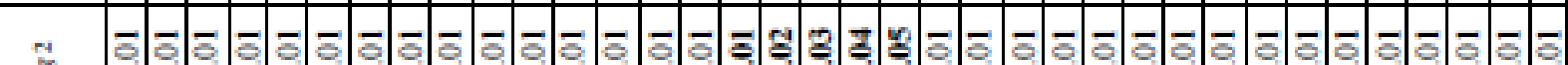

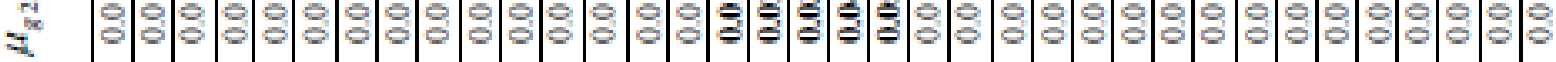

₹

₹

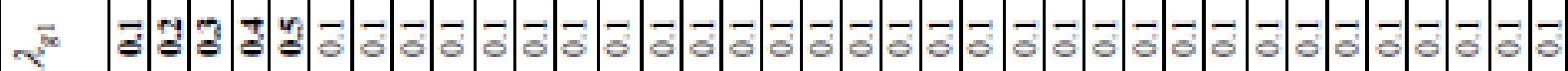




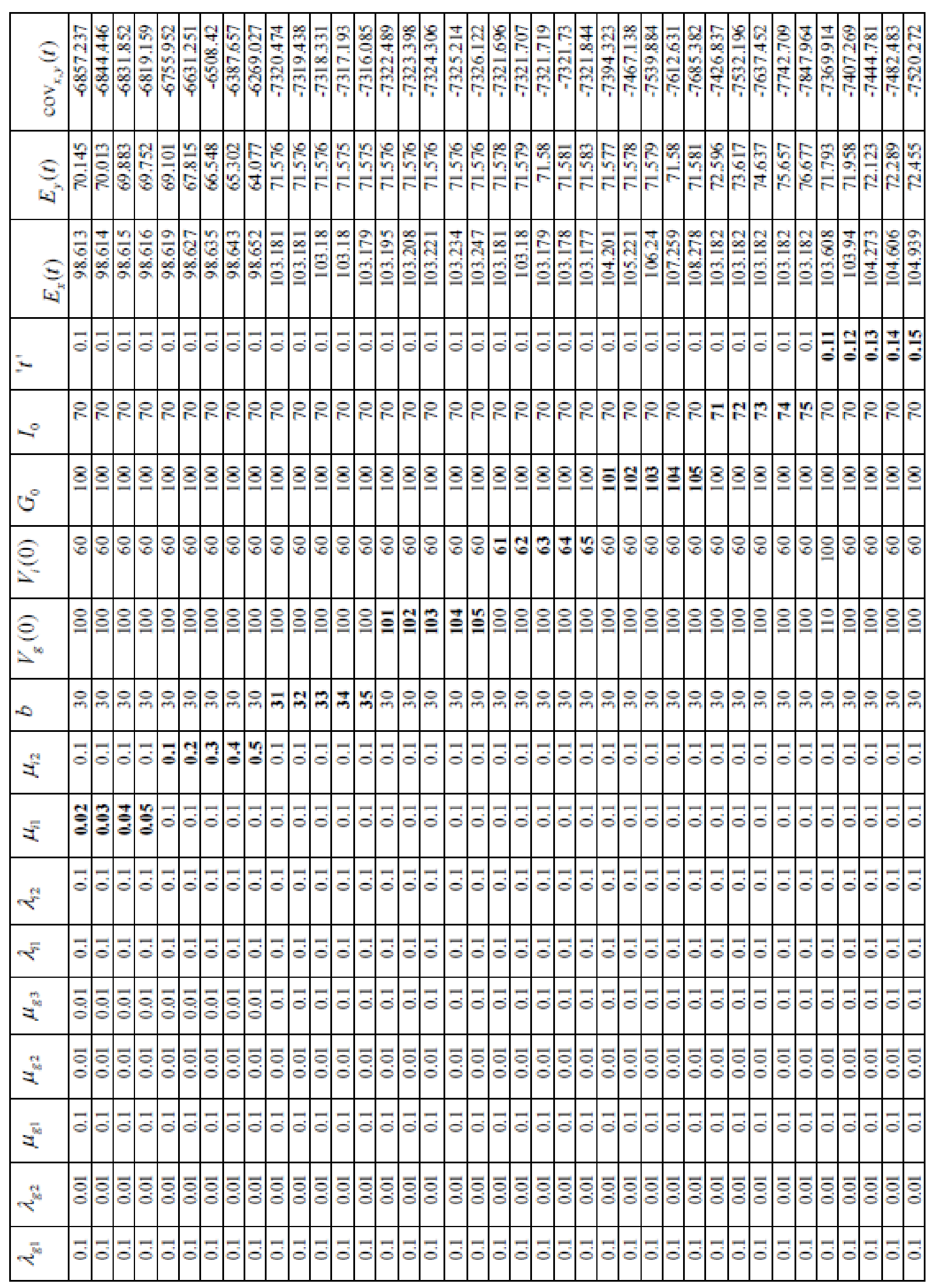




\section{References:}

[1] V.W. Bolie, Coefficients of normal blood glucose regulation, J. Appl. Physiol. 16 (1961) page no.783-788.

[2] Richard N. Bergman, Y. Ziya Ider, Charles R, Bowden, and Claudio Cobelli (1979), Quantitative estimation of insulin sensitivity, American Journal of Physiology-Endocrinology and Metabolism, 236:E667-E677.

[3] Michael E. Fisher (1991), A Semi closed-Loop Algorithm for the Control of Blood Glucose Levels in Diabetics, IEEE Transactions on Biomedical Engineering, Vol. 38, No. I. January 1991, Page No. 57-61.

[4] Paolo Vicini, Andrea Caumo, and Claudio Cobelli (1999), Glucose Effectiveness and Insulin Sensitivity from the Minimal Models: Consequences of undermodeling Assessed by Monte Carlo Simulation.

[5] Andrea De Gaetano and OvideArino (2000), Mathematical modelling of the intravenous glucose tolerance test, Journal of Mathematical Biology 40: Page No. 136-168

[6] Brian Topp, Keith Promislow, Gerda Devries, Robertm. Miuraa and Diane T. Finegood (2000), A Model of $\beta$-Cell Mass, Insulin, and Glucose Kinetics: Pathways to Diabetes, Journal of Theoretical Biology 206, Page No. 605-619.

[7] Athena Makroglou, Jiaxu Li and Yang Kuang (2006), Mathematical models and software tools for the glucose-insulin regulatory system and diabetes: an overview, Applied Numerical Mathematics 56, Page No. 559-573.

[8] P. Tirupathi Rao, K. Srinivasa Rao and C. L. USha (2011), Stochastic Modeling of Blood Glucose Levels in Type-2 Diabetes Mellitus, Asian Journal of Mathematics and Statistics 4 (1): Page No. 56-65.

[9] P. Tirupathi Rao (2012), Stochastic and Optimization Programming for Blood Glucose \& Insulin Regulatory Systems of Type-2 Diabetes, International Journal of Engineering Science and Technology (IJEST), Vol. 4 No.11 November 2012, Page No. 4655-4662. 